\title{
Power Loss and Heat Dissipation Characteristics of High-Power Electromechanical Actuators
}

\author{
Li Peng, Gao Zhi-gang and Zhou Jun \\ Institute of Precision Guidance and Control, School of Astronautics, Northwestern Polytechnical University, 710072, \\ Xi'an, China
}

\begin{abstract}
Due to serious power loss and heating caused by compact volume and high power density of high power electromechanical actuator (EMA), thermal analysis is becoming the key aspects in its design optimization. Numerical calculation method is proposed to calculate the electrical loss, iron core loss and drive chain mechanical loss in EMA. An equivalent thermal network model based on lumped parameters is established, with the acquisition of heat transfer matrix equation of all network nodes. Temperature rise of each part of the EMA is obtained by solving the equation. By comparison of simulated temperature rise in rated operating condition with test results, the accuracy of the model is verified. Then temperature rise simulation of EMA under simulated actual flight condition is carried out, with the results showing positive guidelines in the optimization design of EMA systems.
\end{abstract}

\section{Introduction}

In aerospace field, electromechanical actuator (EMA) is widely used as flight control system to change the attitude of flying vehicles by manipulating the deflection of control surfaces. Due to harsh space limitations and highly dynamic behavior requirements, dimension of EMA is reduced continuously, while the power level is elevating significantly. Therefore, power density and working speed of the servo motor are increasingly high, while its dimension and inertia are cut down to a greatest degree. One problem brought out by this is that power loss and heating of the EMA motor is severe, which has become one of the important factors that limit further improvement of EMA systems [1-4].

Currently, studies on power loss and heat dissipation characteristics of electromechanical servo system focus on the servo motor itself in most cases, while those performing systematic thermal analysis on an actuator can be rarely seen. Furthermore, the influences of transmission mechanism and the actual working condition of actuator system are also not taken into account. Therefore, this paper focuses on the power loss and temperature rise characteristics of EMA from a system perspective, laying a foundation for optimization design and power level improvement of actuator systems.

Research of this work is based on a $4 \mathrm{KW}$ EMA for a primary flight control surface in more electric aircraft. The EMA is composed by a permanent magnet synchronous motor (PMSM), a gear reducer and a high precision potentiometer. A numerical calculation method is used to estimate electrical loss, core loss and mechanical loss of the actuator. Then, temperature field modeling is performed for the system according to an equivalent thermal network method using lumped parameters. In addition, a temperature rise matrix equation is also set up for all nodes in the temperature field. Computational complexity is reduced while the accuracy of the model is guaranteed by this way. At last, system temperature rise simulation and comparative analysis were carried out respectively to the EMA in rated load working condition and simulated flight condition. Measures beneficial to further improvement to the actuator are proposed according to the simulation results.

\section{Analysis on Power Loss of EMA}

Power loss of an EMA is mainly derived from the servo motor and the transmission gear. Specifically, losses incurred by the servo motor include the winding loss and the core loss, and losses of the transmission gear are mainly constituted by the frictional loss of bearings. The power and loss flow of an EMA is presented in Figure 1.

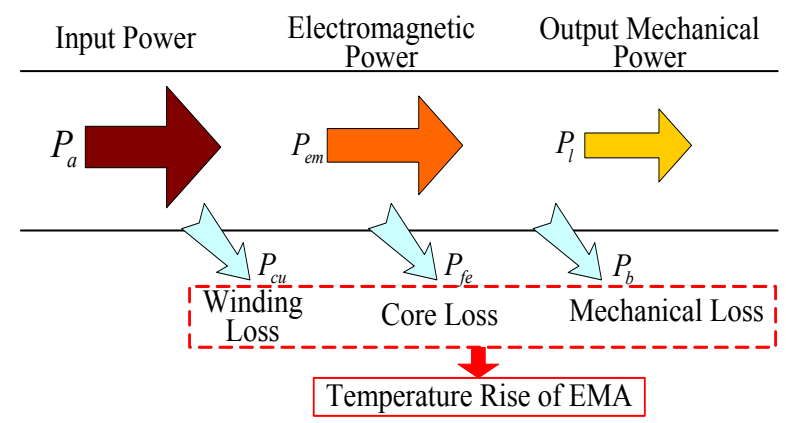

Figure 1. The power flow of EMA. 
Power losses generated in the EMA dissipate in a form of heat, which makes temperature of motor and other mechanism parts rise. Furthermore, temperature rise affects the lifetime and reliability of EMA. Thus, EMA loss and heating modelling aims to check temperature rise situations of all heating parts in a state of rated operating to guarantee that temperature rise of a mechanism within service life does not exceed the permitted value.

\subsection{Servo Motor Loss}

\subsubsection{Winding Loss}

Winding loss, also known as copper loss, is caused by the resistance characteristics of stator winding in PMSM, which can be written as

$$
P_{c u}=N I^{2} R=\rho N I^{2} \frac{L}{A}=\rho N J^{2} L A
$$

where $N$ is the phase number of winding, $I$ is the phase current, $R$ is the phase resistance, $L$ is the length of a phase, $A$ is the cross sectional area of the wire, and $\rho$ is the winding resistivity related with temperature and $\rho=\rho_{20}(1+\alpha(T-20))$, among which $T$ is winding temperature, $\alpha$ is temperature coefficient ( $\alpha=0.0039$ for copper wire) and $\rho_{20}$ is resistivity at $20^{\circ} \mathrm{C}$. Thus, winding loss is related to the material, and directly proportional to both the square of current density and the volume of winding.

As is shown in (1), winding loss depends mainly on the phase current decided by the mechanical load, and is independent of revolving speed. Moreover, winding resistance of high-power motor is relatively small, usually at a magnitude of $\mathrm{m} \Omega$, so the winding loss is usually not the largest part among the losses. However, when a harsh condition is imposed, the winding loss may not be negligible due to its temperature rise.

\subsubsection{Iron Core Loss}

Core loss is one of the major losses in high-power motor and it is especially obvious for multi-pole high-speed motors. Due to the variation of magnetic field inside the motor, magnetic hysteresis loss, eddy current loss and added loss are all incurred in the iron core. Magnetic hysteresis loss is irrelevant with waveforms of the magnetic field but has a direct proportion to amplitude and frequency of the magnetic field. As for the eddycurrent loss, it is not only associated with alternate frequency and amplitude of the magnetic field, but closely related to its waveform.

Magnetic field changes sinusoidally in PMSM. As a result, based on the classical Bertotti model [5-7], core loss can be simplified as:

$$
\begin{aligned}
P_{f e} & =P_{h}+P_{c l}+P_{e x} \\
& =K_{h} f B_{m}^{\alpha}+K_{c l} f^{2} B_{m}^{2}+K_{e x} f^{1.5} B_{m}^{1.5}
\end{aligned}
$$

where $P_{f e}$ is the total core loss of servo motor, $P_{h}, P_{c l}$ and $P_{e x}$ are magnetic hysteresis loss, eddy-current loss and excess loss respectively, $K_{h}, K_{c l}$ and $K_{e x}$ are computing coefficients of magnetic hysteresis loss, eddy-current loss and excess loss, related to both the type and the shape of silicon steel sheet, and they can be obtained by tests. In addition, $\alpha$ is an empirical coefficient equal to $1.6, f$ is the frequency of alternate magnetic field and $B_{m}$ is the magnitude of the magnetic field.

Alternate frequency of the magnetic field within a servo motor increases with the rise of revolving speed, which further leads to rapid growth of core loss. Consequently, temperature rise occurs in the system which cannot be ignored.

\subsection{Mechanical Loss of Transmission Chain}

Mechanical loss is mainly composed of bearing friction loss of the transmission chain. Bearing friction loss is directly proportional to the revolving speed, which takes only a small percent, but it cannot be neglected as far as the high-speed heavy-load mechanism is concerned. Bearing gives rise to friction power loss, which can be expressed as

$$
P_{b}=\sum_{i} T_{i} \omega_{i}
$$

where $P_{b}$ is the total loss of all drive bearings, $T_{i}$ is torque of bearing friction related to bearing structure, lubrication and sealing, and $\omega_{i}$ is the revolving speed of bearing. Heat generated by bearing loss has a direct influence on its service life. Therefore, requirements of bearing load, bearing roller service life and reliability should be taken into the comprehensive consideration at the time of type selection.

\section{Heat dissipation model of EMA}

Temperature field of the actuator was simplified into a thermal network structure with the lumped parameter to perform heat dissipation analysis. The main purpose is to obtain temperature rises of motor winding, core, permanent magnet and the shell. All these parts are heattransfer media with heat sources. The following assumptions are made before calculation [8-11]: (a) all parts inside the mechanism were isothermal bodies of uniform temperature rises, (b) all parts are cooled down naturally, and (c) air temperature of the external environment is constant.

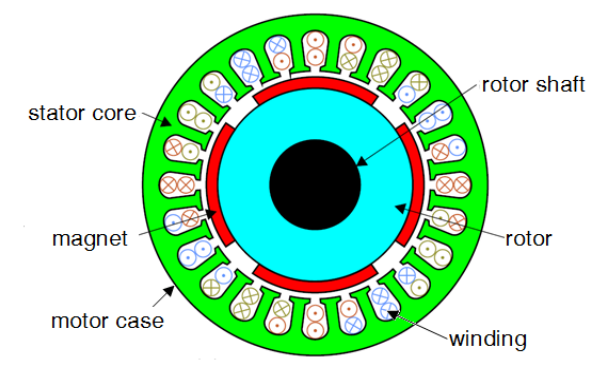

Figure 2. Cross-section of PMSM.

Cross-section structure of the PMSM is shown in Figure 2. Considering the major heat source and loss distribution in the motor as well as the connection between the motor and the reducer, a temperature network model containing 15 nodes is established, as 
shown in Figure 3. Each node represents a main component involved in the heat transfer of the EMA.

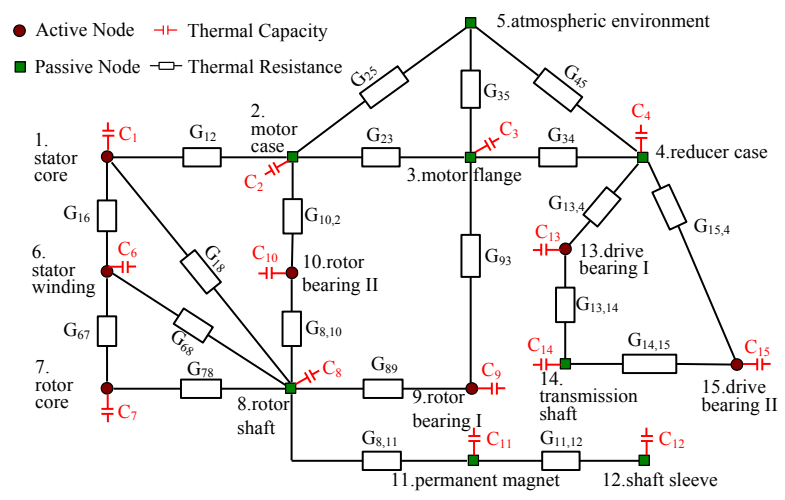

Figure 3. EMA Lumped parameter thermal network model.

Nodes in the temperature network above are adopted according to different characteristics of the main parts of actuator and determined by factors such as temperature distribution, mechanical complexity and material behaviour, etc. All nodes are linked together by thermal conductance $\left(\mathrm{G}_{\mathrm{ij}}\right.$, where $\mathrm{i}$ and $\mathrm{j}$ refer to two nodes adjacent to each other respectively) depending on a thermal conductivity relation[12-15]. Furthermore, the thermal conductivity coefficient is principally related to the material of each node, as is shown in Table 1.

Table 1. Material properties in the EMA thermal model

\begin{tabular}{|c|c|c|c|c|}
\hline Parts & Material & $\begin{array}{c}\text { Density } \\
\left(\mathbf{k g} / \mathbf{m}^{\mathbf{3}}\right)\end{array}$ & $\begin{array}{c}\text { Heat } \\
\text { conductivity } \\
\text { coeff.(W/mK) }\end{array}$ & $\begin{array}{c}\text { Heat } \\
\text { capacity } \\
(\mathbf{J} /(\mathbf{k g} \cdot \mathbf{K}) \mathbf{)}\end{array}$ \\
\hline core & $\begin{array}{c}\text { silicon } \\
\text { steel }\end{array}$ & 7680 & 42.5 & 480 \\
\hline winding & copper & 8900 & 382 & 405.7 \\
\hline $\begin{array}{c}\text { rotor } \\
\text { shaft }\end{array}$ & steel & 7800 & 50 & 460 \\
\hline PM & NdFeB & 7500 & 40 & 480 \\
\hline $\begin{array}{c}\text { rotor } \\
\text { sleeve }\end{array}$ & aluminum & 2700 & 205 & 880 \\
\hline $\begin{array}{c}\text { atmos } \\
\text { phere }\end{array}$ & air & 1.23 & 0.028 & 990 \\
\hline insulation & epoxy & 980 & 0.2 & 1200 \\
\hline
\end{tabular}

Based on heat conduction principle, temperature equation of all nodes in the model can be written as

$$
G T+C \frac{d T}{d t}=Q
$$

where, $T$ is a $n$-dimensional temperature matrix of each node, $G$ is a $n \times n$ dimensional thermal conductivity coefficient matrix of each node, $C$ is a $n \times n$ dimensional heat capacity matrix of each node, $Q$ is a $n$-dimensional heat source matrix, and $\mathrm{n}$ is the number of nodes in the thermal network and $n=15$ in this paper. By solving the above equation, temperature curves for all nodes in the thermal network model can be obtained.

\section{Simulation and Analysis}

Table 2. gives the main parameters of the subject used in simulation. The EMA works in a short-time duty mode with a continuous working time of $5 \mathrm{~min}$ in a rated load condition.

Table 2. Parameters of the EMA model

\begin{tabular}{|c|c|c|c|}
\hline \multicolumn{2}{|c|}{ Servo Motor } & \multicolumn{2}{c|}{ Reducer } \\
\hline mass $(\mathrm{kg})$ & 2.6 & mass $(\mathrm{kg})$ & 3.0 \\
\hline size $(\mathrm{mm})$ & $\Phi 60 \times 110$ & size $(\mathrm{mm})$ & $80 \times 100 \times 150$ \\
\hline rated voltage $(\mathrm{V})$ & 220 & drive ratio & 21 \\
\hline rated power(W) & 4000 & type of drive & gear \\
\hline rated speed(rpm) & 13000 & material & steel \\
\hline pole pairs & 6 & $\begin{array}{c}\text { position } \\
\text { feedback }\end{array}$ & potentiometer \\
\hline $\begin{array}{c}\text { phase } \\
\text { resistance }(\Omega)\end{array}$ & 0.22 & $\begin{array}{c}\text { cooling } \\
\text { pattern }\end{array}$ & passive \\
\hline
\end{tabular}

\subsection{Rated Working Condition Simulation}

Based on the established loss calculation model, loss simulation results for main parts of the EMA in a rated working condition (continuously rotating in rated load) are presented in Figure 4. Core loss of the stator is $358 \mathrm{~W}$ taking the greatest proportion $(61 \%)$, followed by the winding loss (34\%) and the mechanical loss takes only $1 \%$ at the minimum level.

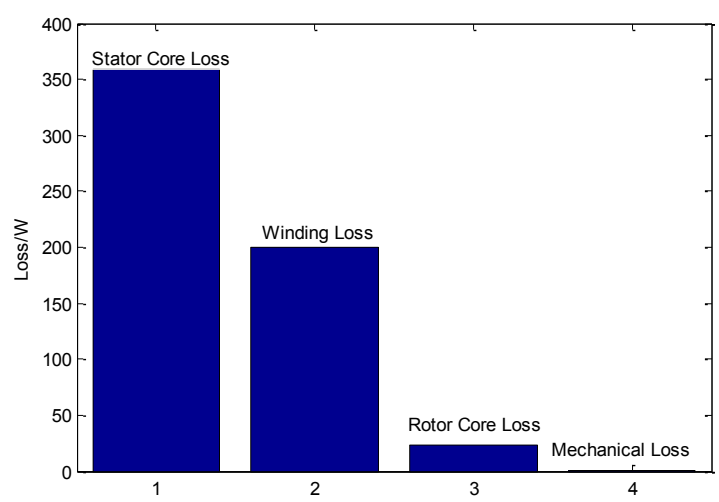

Figure 4. Power loss in rated working condition.

Figure 5 shows temperature rise simulation curves for all major parts inside the actuator, where the ambient temperature is set at $300 \mathrm{~K}$ or $26.85^{\circ} \mathrm{C}$. At $300 \mathrm{~s}$, sequencing of temperature rise values are winding $>$ stator core $>$ shell $>$ rotor. Specifically, temperature rises of the winding and the shell are $81^{\circ} \mathrm{C}$ and $59^{\circ} \mathrm{C}$ respectively.
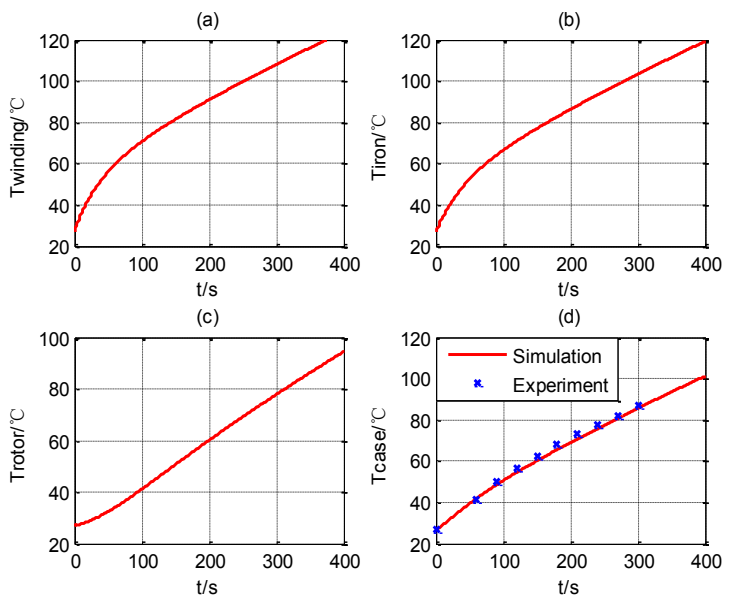

Figure 5. Simulated temperature rise and test result in rated working condition. 
In Figure 5(d), the measured temperature curve of shell is compared with the simulation curve, which clearly signifies that both curves highly coincide with each other. Initial temperature of the test is $26.6^{\circ} \mathrm{C}$ and temperature rise reaches $60.2^{\circ} \mathrm{C}$ at 300s. Thus the accuracy of the heat dissipation model is verified. Figure 6 gives the thermograph of the mechanism when the test ended at $300 \mathrm{~s}$, with the hottest spot of $86.8^{\circ} \mathrm{C}$.

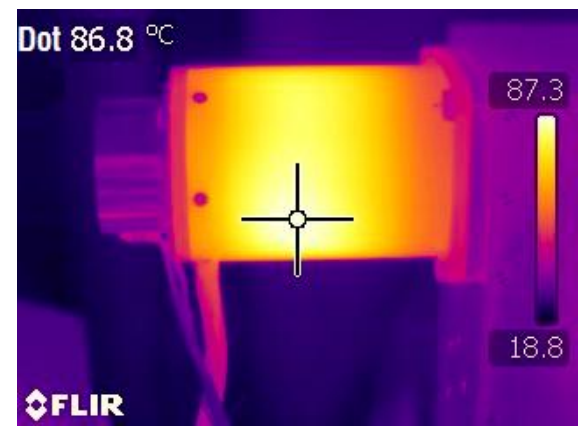

Figure 6. Thermograph of EMA in rated working condition.

Figure 7 displays the comparison of temperature rise curves between an actuator (with reducer) and a single motor (without reducer) under identical rated load circumstances. It can be found that temperature rising in the actuator is slower than a single motor due to heat capacity and heat dissipation effects of the transmission devices in the actuator. At 300 s, average temperature rise of each part of the actuator is about $30^{\circ} \mathrm{C}$ lower than that of the motor.
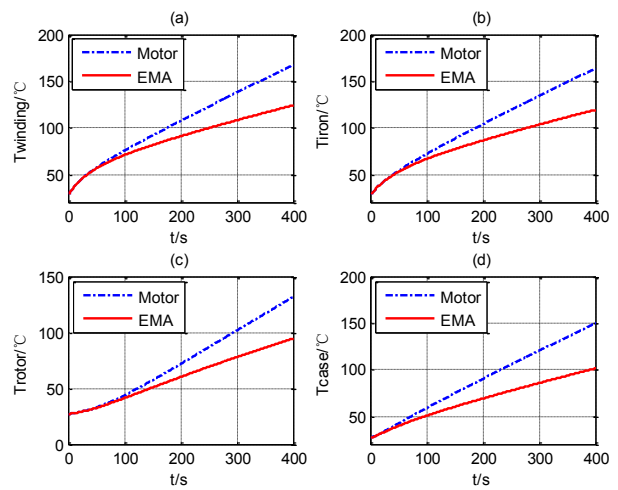

Figure 7. Motor and EMA temperature rise comparison in rated working condition.

\subsection{Flight condition simulation}

During actual flight mission, the EMA does not entirely work in rated load state. So it is necessary to perform simulation analysis on practical situations. A "three-stage process" approach is proposed in this paper to simulate general flight characteristics. In detail, it consisted of low-frequency sinusoidal motion, high-frequency sinusoidal motion and step motion; and each of them occupies a third segment of the whole flight time. Position command signals sent to the actuator are as follows: $\mathrm{u}=10 \sin (0.4 \pi \mathrm{t})$ for $0 \sim 100 \mathrm{~s}, \mathrm{u}=\sin (20 \pi \mathrm{t})$ for $100 \sim 200 \mathrm{~s}, \mathrm{u}=10 \operatorname{square}(0.4 \pi \mathrm{t})$ for $200 \sim 300 \mathrm{~s}$ and $\mathrm{u}=0$ for $300 \sim 400$ s. Besides, unit of the command is $\mathrm{V}$ and the corresponding rudder coefficient is $2 \% / \mathrm{V}$. Position command and actuator position feedback curves are given in Figure 8(a).
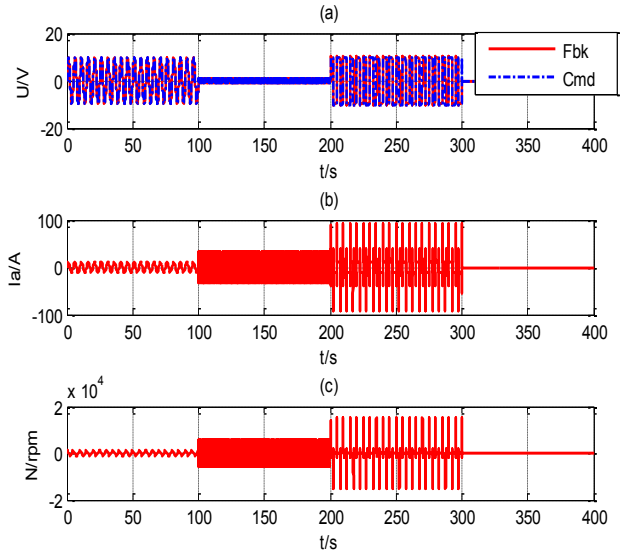

Figure 8. Motor current and velocity in simulated flight condition

Figure $8(\mathrm{~b})$ and $8(\mathrm{c})$ represent the bus current of the actuator and the revolving speed of the motor under the given command respectively. It can be seen that at a lowfrequency stage of $0 \sim 100$ s, both current and revolving speed of the actuator are very low, at a high-frequency stage of $100 \sim 200$ s, current rises up at a peak of about $40 \mathrm{~A}$ and the revolving speed also increases, at a step motion stage of 200 300s, current surge approaches $100 \mathrm{~A}$ in each cycle and the corresponding revolving speed also reaches about $16,000 \mathrm{rpm}$ in transient process.
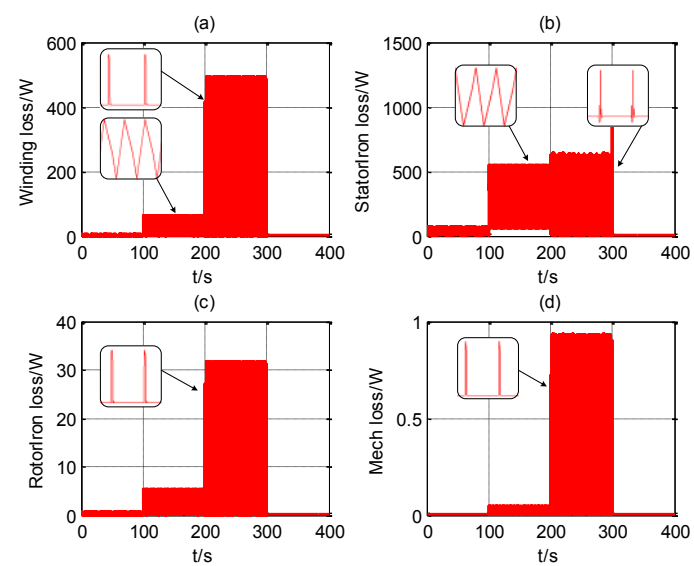

Figure 9. Loss curve of EMA in simulated flight condition

Figure 9 represents loss curves of the main parts in the actuator under the given commands. Clearly, loss curve characteristics are in consistence with the variation features of current and motor's revolving speed shown in Figure 8.

Temperature rise curves of the main parts of EMA in a simulated flight condition are shown in Figure 10. At the low-frequency stage of $0 \sim 100 \mathrm{~s}$, temperature rises of all parts in the actuator are very low. At the highfrequency stage of 100 200s, rapid temperature rise is incurred by large current, high speed and great average loss, and this is also a primary stage of temperature rise. At the step motion stage of 200 300s, the average loss is not high although it has a high peak power loss. Gradual heat dissipation leads to decreases in temperature of winding and core, while temperature of rotor and shell 
still rises by $5^{\circ} \mathrm{C}$ and $1.5^{\circ} \mathrm{C}$ respectively. At $300 \mathrm{~s}$, temperature rise of winding parts arrives at the maximum value of $30^{\circ} \mathrm{C}$, which is only $37 \%$ of that in a rated working condition.
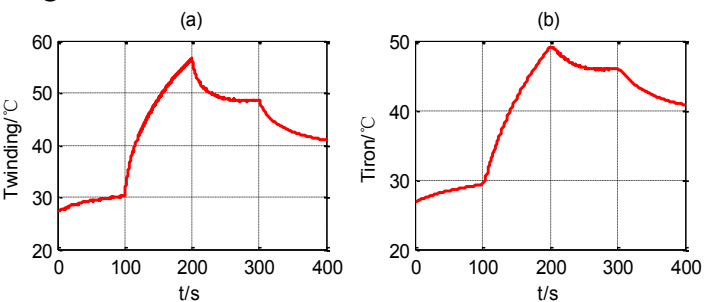

(c)

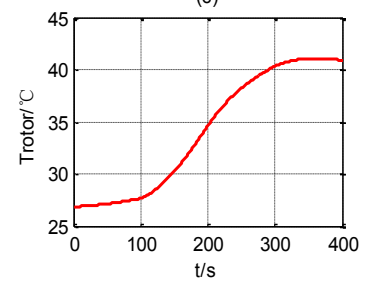

(d)

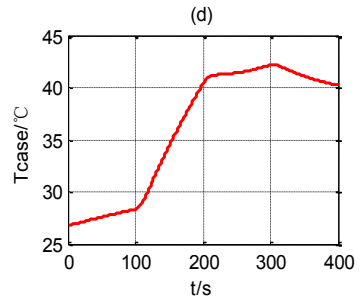

Figure 10. EMA temperature rise in simulated flight condition.

\section{Conclusion}

Aiming at severe loss and heating problem of high-power electromechanical actuators, a thermal analysis approach based on equivalent thermal network method with lumped parameters is proposed in this paper. An equivalent thermal network model is constructed based on a numerical calculation method to precisely estimate actuator losses. Thermal equilibrium matrix equation is established for all nodes in the thermal network to obtain temperature rise curves of all main parts in the actuator in rated load condition and simulated flight condition.

According to simulation results, it can be concluded that: (a) transmission mechanism of the actuator is able to substantially improve temperature rise of the EMA due to its heat capacity and heat dissipation effects, despite that it incurs a part of mechanical loss, (b) integrated design for servo motor and transmission mechanism shell can further enhance thermal characteristics of the system, and (c) actual operation mode of EMA has a dramatic effect on it temperature rise, thus the thermal analysis should be performed for the actual flight course so as to optimize the design of the actuators.

\section{Acknowledgement}

This work was supported by the Fundamental Research Funds for the Central Universities (No.3102017GX14003) of China.

\section{References}

1. Chen Yiguang, Zheng Jun, Wei Juan, et al. Design of PMSM for actuator and its temperature field analysis[J]. Transactions of China Electrotechnical Society, 30(14): 94-99, (2015).

2. Takeda Y, Takahashi Y, Fujiwara K, et al. Iron Loss
Estimation Method for Rotating Machines Taking Account of Hysteretic Property[J]. Magnetics IEEE Transactions on, 51(3):1-4, (2015).

3. Wang W, Zhou Y, Chen Y. Investigation of lumpedparameter thermal model and thermal parameters test for IPMSM[C]. International Conference on Electrical Machines and Systems, IEEE, (2015).

4. Zhang Jianzhong, Jiang Yongjiang. Thermal Analysis of Constant Frequency Double Rotor Permanent Magnet Generator Based on Equivalent Thermal Network Method. Transactions of China Electrotechnical Society. 30(02): 87-97, (2015).

5. Kong Xiaoguang, Wang Fengxiang, Xing Junqiang. Losses calculation and temperature field analysis of high speed permanent magnet machines[J]. Transactions of China Electrotechnical Society, 27(9): 166-172, (2012).

6. Zheng D, Wang D, Li S, et al. Eddy current loss calculation and thermal analysis of axial-flux permanent magnet couplers[J]. Aip Advances, 7(2):24-35, (2017).

7. DONG Jianning, HUANG Yunkai, JIN Long, et al. Review on High Speed Permanent Magnet Machines Including Design and Analysis Technologies[J]. Proceedings of the Chinese Society for Electrical Engineering, (27): 4640-4653, (2014).

8. LI Liyi, ZHANG Jiangpeng, YAN Haiyuan, et al. Study on the Optimization of Thermal Conductivity and 3D Temperature Filed Calculation for the High Power Density Motor[J]. Proceedings of the Chinese Society for Electrical Engineering, 36(13): 3642-3650, (2016).

9. Woodburn D, Wu T F, Zhou L, et al. High-Performance Electromechanical Actuator Dynamic Heat Generation Modeling[J]. IEEE Transactions on Aerospace \& Electronic Systems, 50(1):530-541, (2014).

10. Sefkat G. Investigating static and dynamic characteristics of electromechanical actuators (EMA) with MATLAB GUIs[J]. Computer Applications in Engineering Education, 18(2):383-396, (2009).

11. Bahman A S, Ma K, Blaabjerg F. A Lumped Thermal Model Including Thermal Coupling and Thermal Boundary Conditions for High Power IGBT Modules[J]. IEEE Transactions on Power Electronics, PP(99):1-14, (2017).

12. Kim K S, Lee B H, Hong J P. Improvement of Thermal Equivalent Circuit Network and Prediction on Heat Characteristic of Motor by Calculation of Convection Heat Transfer Coefficient[C]. The 6th International Conference on Electromagnetic Field Problems and Applications, IEEE, (2012).

13. Boglietti A, Carpaneto E, Cossale M, et al. StatorWinding Thermal Models for Short-Time Thermal Transients: Definition and Validation[J]. IEEE Transactions on Industrial Electronics, 63(5): 2713-2721, (2016).

14. Ahmed F, Ghosh E, Kar N C. Transient thermal analysis of a copper rotor induction motor using a lumped parameter temperature network model[C], IEEE Transportation Electrification Conference and Expo, IEEE, (2016).

15. Sciascera C, Giangrande P, Papini L, et al. Analytical Thermal Model for Fast Stator Winding Temperature Prediction[J]. IEEE Transactions on Industrial Electronics, 64(8): 6116-6126, (2017). 